\title{
Analysis of Broadband Piezoelectric Transducers by Discrete Time Model
}

\author{
M.G.S. Ali \\ Physics Department, Faculty of Science, Minia University, Egypt.
}

The simulations of a discrete time model of the impulse response of matched and backed piezoelectric transducers have been used to design sensitive short-response transducers. The effect of matching and backing materials layers on the performance of piezoelectric transducers working into water load is investigated. The optimum acoustical characteristics of the quarter-wave length matching layers are determined relatively to a compromise between the sensitivity and the bandwidth. 


\section{Introduction}

In ultrasonic nondestructive tests and ultrasonic diagnosis, it is necessary to transmit and detect short ultrasonic pulses to attain good range of resolution. The usual transducer generates ultrasonic pulses of pulse width much longer than electrical signals. For damping this free oscillation, acousticbacking material is used at the back of the transducer but the backing materials absorb acoustic energy and therefore reduce the transducer sensitivity [1].

Transducer sensitivity and bandwidth are generally improved by adding single or multiple quarter -wave length matching layers between the piezoelectric transducer and the load medium and this in turns reduce losses between the transducer and load. In such cases, the acoustic impedance of selected the matching layers is known to act as an important factor in the performance of the transducer. Several theoretical methods for analyzing the optimum mechanical impedance of the intermediate layer, which provides broad frequency band, have been reported in the literature [2-5].

This paper describes the use of a time domain model of the thickness of piezoelectric transducer for the estimation of the best matching and backing layers.

The model is implemented directly in the time domain, in the form of a recursive digital filter [1,2]. The optimum acoustical characteristics of the matching layers and backing block are determined by defining a compromise between the sensitivity and the bandwidth of the impulse spectra.

\section{Transducer equivalent circuits}

The transducer performs the conversion of electrical energy into mechanical energy, and conversely, the conversion of mechanical energy into electrical energy. There is a fundamental relationship between the properties of the transducer and the techniques have been developed for modeling transducer behaviour and hence predicting the required system response.

There are many computer models describing the behaviour of ultrasonic piezoelectric transducers [6,7]. In this paper, the simulation was applied using a previously developed digital computer model [1] to evaluate the discrete time responses. The model including z-transform techniques [8] to provide a discrete time implementation of the original frequency domain equivalent circuit of Berlincourt et al. [9]. By use of a simple algorithm which describes the acoustic impedance looking into an arbitrary number of lose less 
acoustic transmission lines and the transfer of force across such a system [2], together with the use of Thevenin's theorem, the model has been extended to incorporate any number of matching and backing layers and any arbitrary electrical network. The computer model is split into two parts. First, the basic transfer function of the loaded piezoelectric element is evaluated in discrete form using the z-transform technique. Secondly, the discrete time responses so obtained are then processed by a nested set of digital filter operations that simulate reverberation in the coupling layers connected to the transducer and backing and load media.

Three transfer functions are required for the transducer whose equivalent circuit is depicted in Fig. (1). The voltage transfer function, relating the Thevenin equivalent of the excitation voltage $\mathrm{V}_{\mathrm{ET}}$ to the voltage developed across the transducer terminals $(\mathrm{V})$ can be written as,

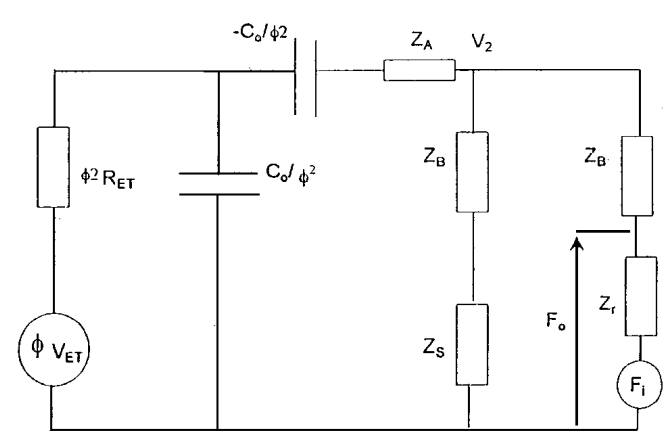

Fig.(1) Equivalent circuit of the piezoelectric transducer.

$$
\begin{aligned}
& V=\frac{V_{E T}\left(s C_{0} R_{M}-\phi^{2}\right)}{s C_{0}\left(1+s C_{0} R_{E T}\right) R_{M}-\phi^{2}} \\
& R_{M}=\frac{2 Z_{A} Z_{B}+Z_{B}^{2}+\left(Z_{A}+Z_{B}\right)\left(Z_{r}+Z_{s}\right)+Z_{r} Z_{s}}{2 Z_{B}+Z_{r}+Z_{s}},
\end{aligned}
$$

Where $\mathrm{C}_{0}$ is the static capacitance of the piezoelectric element, and $\mathrm{s}$ is the Laplace variable. $\Phi$ represents the electrical to mechanical conversion factor. $Z_{\mathrm{s}}$ and $Z_{r}$ represents the distributed mechanical impedance at the back and front face of the piezoelectric element respectively. $Z_{A}$ and $Z_{B}$ represent the distributed components of the familiar ' $T$ ' equivalent circuit of a transmission line.

The calculation of the transmit and receive transfer functions involves a two-step procedure. For the transmit response, first the response at the point labeled $\mathrm{V}_{2}$, Fig.(1), is obtained. Next the transmitted force across the matching layers is calculated from the response at $\mathrm{V}_{2}$. On reception, the force transfer across an arbitrary number of matching layers can be written as,

$$
V=\frac{\left\{s C_{0}\left(1+s C_{0} R_{E T}\right) Z_{A}-\phi^{2}\right\}\left(Z_{B}+Z_{S}\right) F_{i}}{s C_{0}\left(1+s C_{0} R_{E T}\right)\left\{2 Z_{A} Z_{B}+Z_{B}{ }^{2}+Z_{S}\left(Z_{A}+Z_{B}\right)\right\}-\phi^{2}\left(2 Z_{B}+Z_{S}\right)}
$$


Eqs.(1) and (2) represented in terms of discrete time, a discrete

$$
S=\frac{2}{T}\left\{\frac{1-z^{-1}}{1+z^{-1}}\right\}
$$

approximation to the Laplace variable $\mathrm{s}$ has to be incorporated into this

expression. There are numerous discrete time representations available to approximate the differential operator $\mathrm{s}$, however, due to the extensive use of cascade programming techniques used

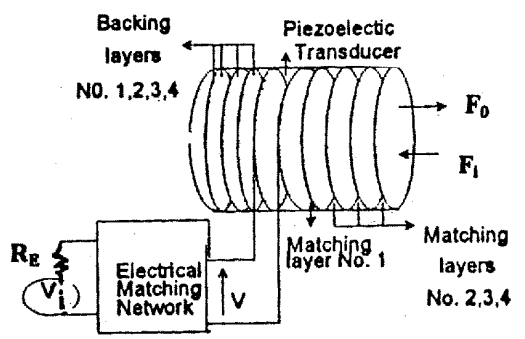

Fig.(2) Schematic diagram of the model. in this model, a fixed structure approximation for $\mathrm{s}$ is recommended. The bilinear transform maps continuous functions of $\mathrm{s}$ into z-plane is given by

The full model is represented in schematic form, Fig.(2).

\section{Simulation Results}

The simulation was applied using typical data for transducers constructed with a lead Zirconate-titanate (PZT-5A) piezoelectric element [9] with different backing blocks and different quarter-wave length matching layers of materials. The transmitter response was obtained for a $10-\mathrm{mm}$ diameter pulse-echo transducer of $5 \mathrm{MHz}$ center frequency, and radiating into water medium. Assuming simple impulse excitation to the transducer, and consider the received echo waveform and its frequency spectrum, at the terminals of the same transducer. The behaviour of the transducer was simulated for a different quarter matching layers at its front face with light, medium and heavy damping applied to the back face.

The sensitivity is defined as the maximum height of received pulse, given in terms of volt. Fig.(3a) shows the expected waveform at the transducer terminals when the device acts as a receiver with no matching layer and zero backed transducer. Fig.(3b) is a repeat of the simulation of Fig.(3a) but with a crown glass backing block of acoustic impedance $11.4 \times 10^{6} \mathrm{~kg} \mathrm{~m}^{-2} \mathrm{~s}^{-1}$. Fig.(3c) shows the same simulation response for a PZT-5A transducer loaded by a tungsten-epoxy [2] $\left(\mathrm{z}=19 \times 10^{6} \mathrm{~kg} \mathrm{~m}^{-2} \mathrm{~s}^{-1}\right)$ backing block. Fig.(3d) is a repeat of the simulation of Fig.(3a) but with a backing block of acoustic impedance equal to the acoustic impedance of the transducer $\left(33.75 \times 10^{6} \mathrm{~kg} \mathrm{~m}^{-2} \mathrm{~s}^{-1}\right)$. Once the impulse responses were obtained, a Fast Fourier Transform was performed on the data. Figs.(4a-d) shows the pulse-echo transducer frequency responses for 
the four different backings described above respectively. From Figs.(3a-d), it can be seen that the sensitivity decreased from 2 to 0.35 volt and the half amplitude bandwidth increased from 0.58 to $2.5 \mathrm{MHz}$ in Figs.(4a-d).In practice, there is a compromise in relative peak sensitivity, which fall approximately proportionally, as the bandwidth is increased.
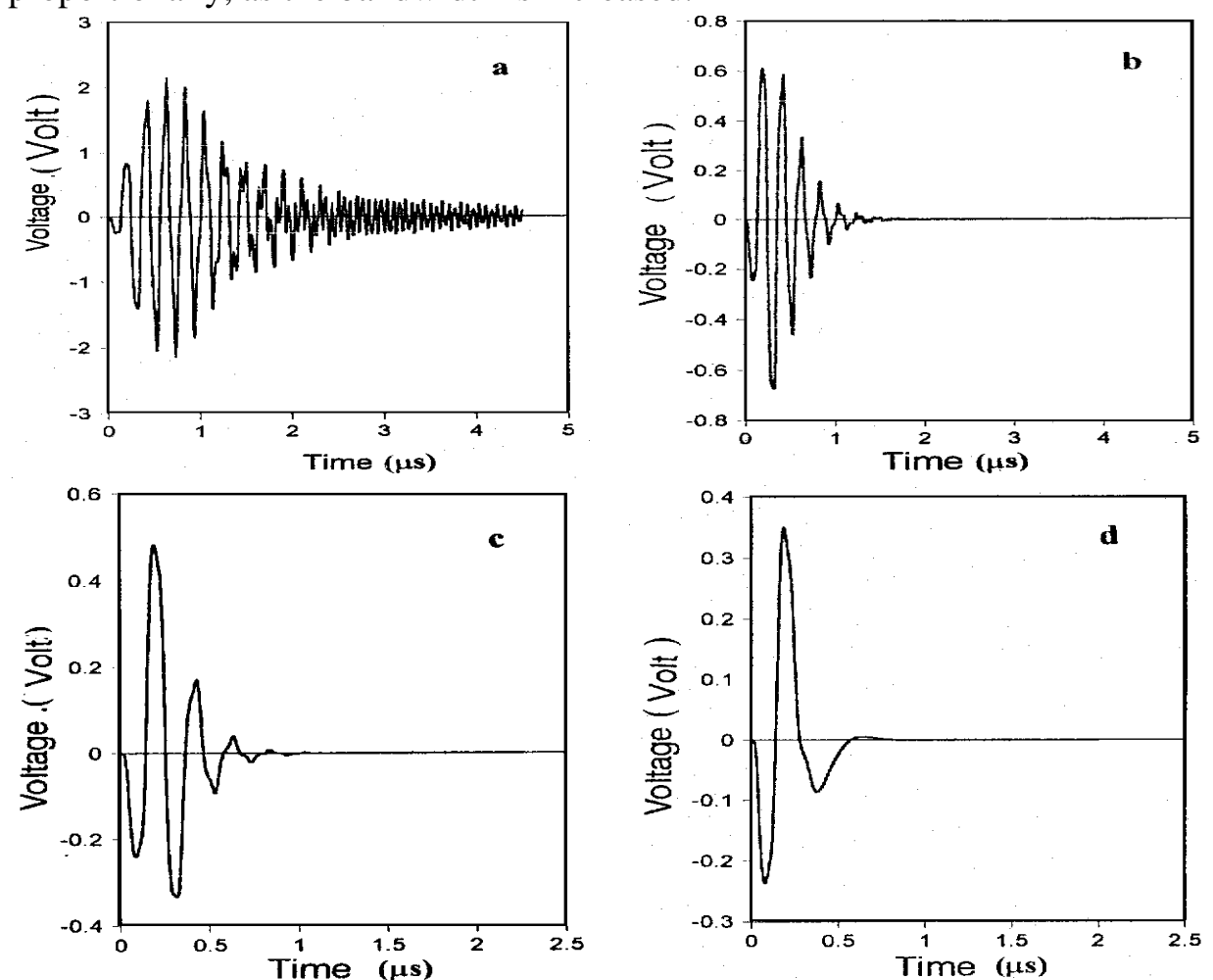

Fig.(3) The pulse-echo transducer responses with no matching layer operating into water load.(a) zero backing, (b) backing of crown glass, (c) backing of tungsten-epoxy, (d) backing block of acoustic impedance equal to the impedance of the transducer.

Let us now examine changes in the performance of transducers with two, three and four quarter wavelength matching layers. Varying each of the matching layer materials and searched for the combination of materials, that gives a wide bandwidth. The optimum result for two matching layers can be obtained by using glass arsenic trisulphide $[10]\left(\mathrm{z}_{1}=8 \times 10^{6} \mathrm{~kg} \mathrm{~m}^{-2} \mathrm{~s}^{-1}\right)$ for the first matching layer and a polystyrene $[11]\left(\mathrm{z}_{2}=2.5 \times 10^{6} \mathrm{~kg} \mathrm{~m}^{-2} \mathrm{~s}^{-1}\right)$ for the second matching layer. Desilets et al [12] had described a generalized formulation for several front matching layers. From their analysis, the acoustic impedance of a two matching layers on the front of the transducer should have a value of $\mathrm{z}_{1}=$ $\mathrm{z}_{\mathrm{T}}^{4 / 7} \mathrm{Z}_{\mathrm{M}}^{3 / 2}=8.6 \times 10^{6} \mathrm{~kg} \mathrm{~m}^{-2} \mathrm{~s}^{-1}$ and $\mathrm{z}_{2}=\mathrm{z}_{\mathrm{T}}^{1 / 7} \mathrm{z}_{\mathrm{M}}^{6 / 7}=2.3 \times 10^{6} \mathrm{~kg} \mathrm{~m}^{-2} \mathrm{~s}^{-1}$ where $\mathrm{z}_{\mathrm{T}}$ 
and $\mathrm{z}_{\mathrm{M}}$ are the acoustic impedance of the transducer and the load medium respectively. This result is very close to our optimal configurations.
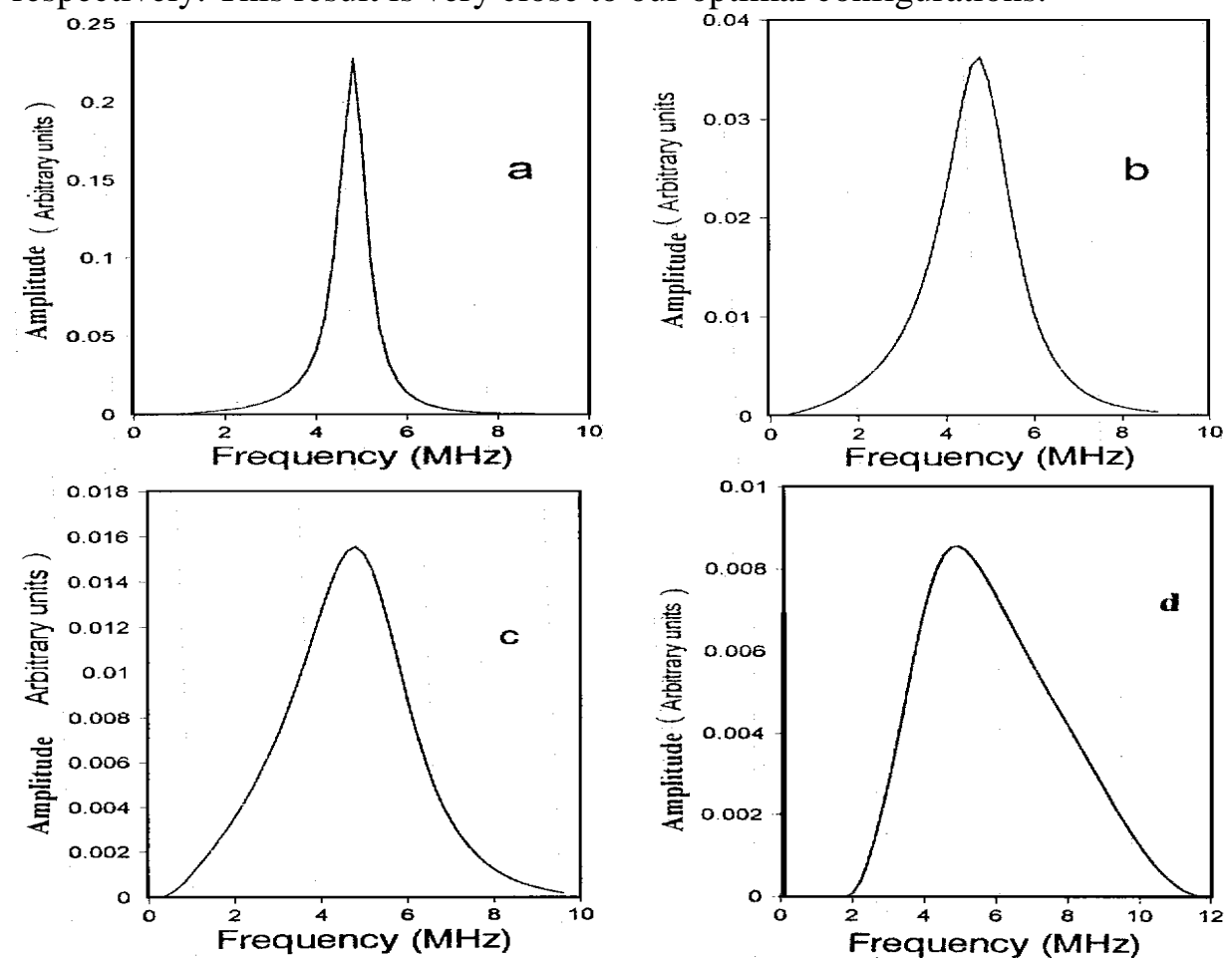

Fig.(4) Pulse-echo frequency responses for four different transducer backings as shown in Fig.(3).

The optimum condition for the three matching layers can be obtained by using tungsten epoxy [13] $\left(\mathrm{z}_{1}=10 \times 10^{6} \mathrm{~kg} \mathrm{~m}^{-2} \mathrm{~s}^{-1}\right)$ for the first matching layer, polmethylmeth-acrylate [10] $\left(\mathrm{z}_{2}=3.2 \times 10^{6} \mathrm{~kg} \mathrm{~m}^{-2} \mathrm{~s}^{-1}\right)$ for the second matching layer and a mathylplaten [10] $\left(\mathrm{z}_{3}=1.85 \times 10^{6} \mathrm{~kg} \mathrm{~m}^{-2} \mathrm{~s}^{-1}\right)$ for the third matching layer.

Also, four matching layers can be obtained by using glass $\left(\mathrm{z}_{1}=11.4 \times 10^{6} \mathrm{~kg} \mathrm{~m}\right.$ ${ }^{2} \mathrm{~s}^{-1}$ ) for the first matching layer, glass beads and epoxy [11] $\left(\mathrm{z}_{2}=4.2 \times 10^{6} \mathrm{~kg} \mathrm{~m}^{-2} \mathrm{~s}^{-}\right.$ $\left.{ }^{1}\right)$ for the second matching layer, a polystyrene [11] $\left(\mathrm{z}_{3}=2.5 \times 10^{6} \mathrm{~kg} \mathrm{~m}^{-2} \mathrm{~s}^{-1}\right)$ for the third matching layer and a mathylplaten [10] $\left(\mathrm{z}_{4}=1.85 \times 10^{6} \mathrm{~kg} \mathrm{~m}^{-2} \mathrm{~s}^{-}\right.$ $\left.{ }^{1}\right)$ for the four matching layer. Fig.(5) is a repeat of the simulation of Fig.(4a) but with the optimum condition for two, three and four matching layers described above.

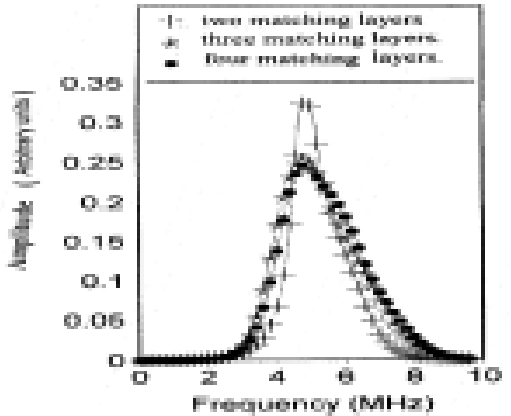

Fig.(5) Pulse-echo frequency response of Fig.(3a). 
It is clear from this figure that the four matching layers made a little change in the bandwidth and sensitivity with respect to the three matching layers. Fig.(6) shows the received waveforms computed for the two and three matching layers. It is clear from these figures that the sensitivity and the magnitude of bandwidth of frequency spectrum are strongly dependent on the number of matching layers. Fig.(7) shows the same simulation of Fig.(3b) but with two and three matching layers. The spectrum of Fig.(7) is shown in Fig.(8). Fig.(9) is a repeat of the simulation of Fig.(3c) but with two and three matching layers. Fig.(10) shows the frequency spectrum of Fig.(9). These results confirm that the transducer backing and acoustic impedance connected to it dictates the bandwidth and sensitivity of the transducer. Fig.(11) shows the variation of sensitivity as functions of backings impedance at different matching layers. Fig(12) shows the variation of half amplitude bandwidth as functions of backings impedance at different matching layers. Our results show that the sensitivity and bandwidth increase with increases the number of matching layers. The backing impedance decreases the sensitivity and the bandwidth increase. These results can be used to careful transducer design to arrive at an adequate compromise between bandwidth and sensitivity.
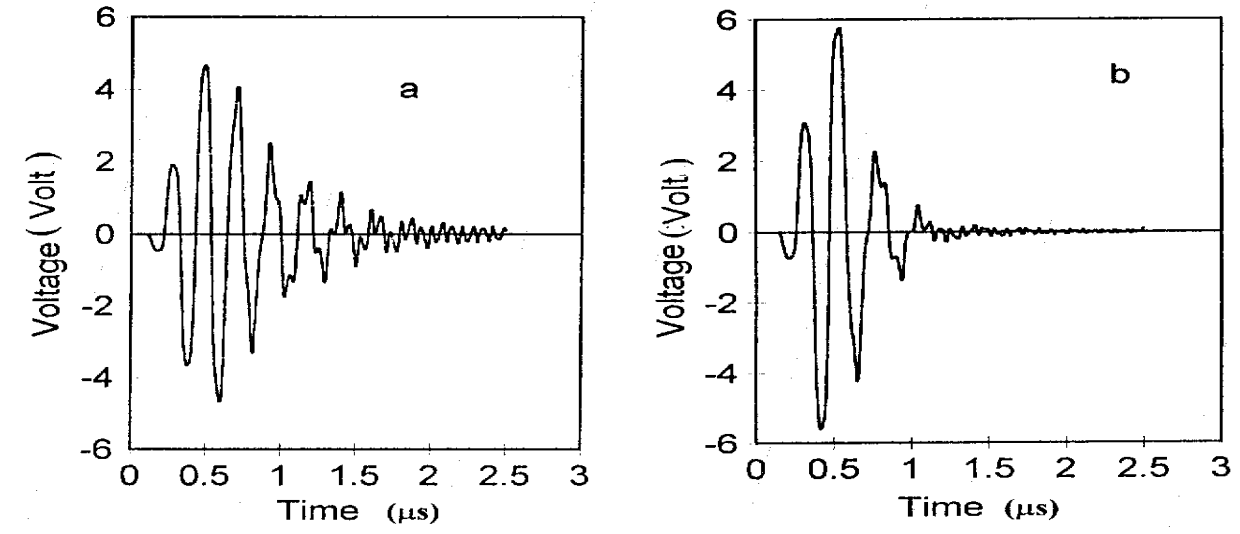

Fig.(6) Received waveforms of Fig.(3a) with (a) two matching layers, (b) three matching layers.
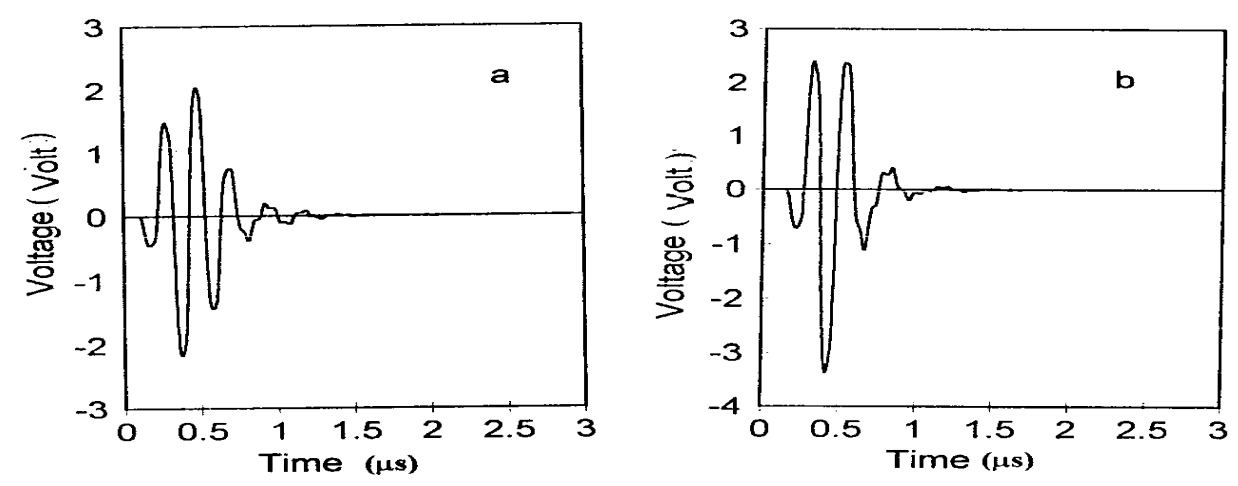

Fig.(7) The pulse-echo response of Fig.(3b) with (a) two matching layers (b) three matching layers. 


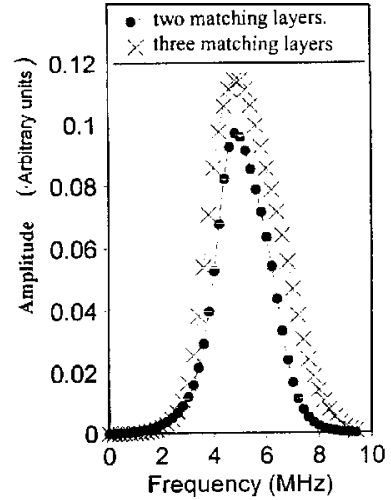

Fig.(8) Amplitude frequency spectrum of Fig.(7).

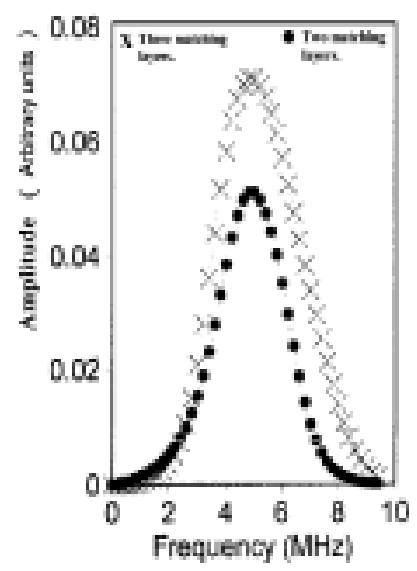

Fig.(10)

Amplitude frequency spectrum of Fig.(9).
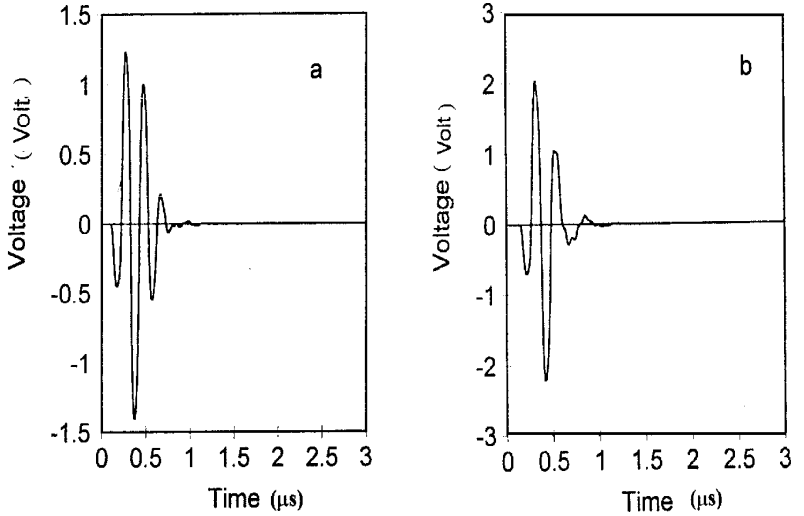

Fig.(9) The pulse-echo response of Fig.(3c) with (a) two matching layers (b) three matching layers.

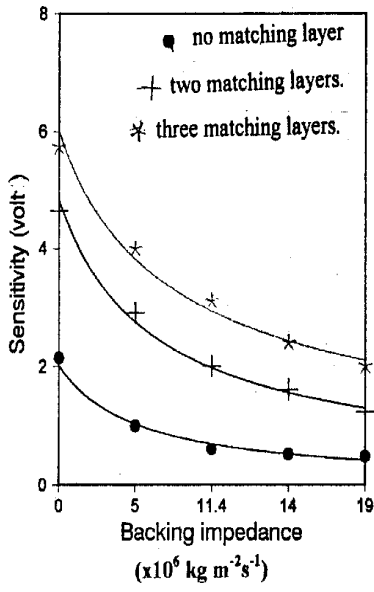

Fig.(11)

Dependence of sensitivity on the backing acoustic impedance.

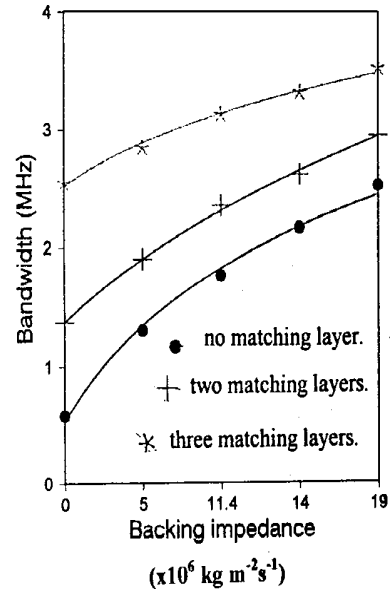

Fig.(12)

Dependence of half amplitude bandwidth on the backing acoustic impedance.

\section{Conclusions}

A discrete time model of the thickness-mode piezoelectric transducer has been used to predict the influence of mechanical layers at its front face with light, medium and heavy damping applied to the back face. The model is obtained from the analysis of the electrical equivalent circuit of a piezoelectric device. The received waveform and frequency response of ultrasonic transducer with two, three and four quarter wavelength acoustic matching layers have been analyzed, and found that the response of the transducer appears to improve as 
the number of matching layers increases. The trade-off between sensitivity and bandwidth has been developed. The technique could be extended to incorporate the effect of absorption for backing block.

\section{References}

1. M.G.S.Ali and A.R.Mohamed, Ultrasonic, 30(5), 311, (1992).

2. M.G.S.Ali, J.Sound and Vibration, 224(2), 349, (1999).

3. M. Redwood, Applied Materials Research, 2, 76, (1963).

4. G.K. Lewis, Acoustic Imaging, 8, 395, (1979).

5. K.Yamaguchi, H. Yagami and T. Fujii, IEEE Trans. Ultrason. Ferroelectric, Freq. Contr., UFFC-33(6), 669, (1986).

6. R.E.Challis and J.A. Harrison, J. Acoust Soc. Am., 74, 1673, (1983).

7. G.Hayward, C.J.Macleod and T.S.Durrani, J.Acoust. Soc. Am., 76, 369, (1984).

8. E.I. Jury, Theory and application of the z-transform method, Wiley, New York, (1964).

9. D.A. Berlincourt, D.R. Curran, and H. Jaffe, Piezoelectric and piezomagnetic materials and their function in transducers, in: Physical Acoustics IA (Ed Mason W.P.) Academic, New York (1964).

10. G.W.C. Kaye and T.H. Laby, Tables of physical and chemical constants, London, England: Longmans, 1968.

11. J.W. Hunt, M. Akditi and F.S. Foster, IEEE Trabs Bio. Eng., 30, 454, (1983).

12. C.S. Desilets, J.D. Fraser and G.S. Kino, IEEE Trans. Sonics Ultrason., Vol.SU-25, 115-125, (1978).

13. G. Kossof, The effects of backing and matching on the performance of piezoelectric ceramic transducers, IEEE Trans, Sonics Ultrason.Vol. SU13, 20-30, (1966). 